\title{
Estimating Driving Performance Based on EEG Spectrum Analysis
}

\author{
Chin-Teng Lin \\ Brain Research Center, University System of Taiwan, Taipei 112, Taiwan \\ Department of Electrical and Control Engineering, National Chiao-Tung University, Hsinchu 300, Taiwan \\ Email:ctlin@mail.nctu.edu.tw \\ Ruei-Cheng Wu \\ Brain Research Center, University System of Taiwan, Taipei 112, Taiwan \\ Department of Electrical and Control Engineering, National Chiao-Tung University, Hsinchu 300, Taiwan \\ Email:allen.ece86g@nctu.edu.tw
}

\section{Tzyy-Ping Jung}

Institute for Neural Computation, University of California, San Diego, La Jolla, CA 92093-0523, USA

Email:jung@sccn.ucsd.edu

\author{
Sheng-Fu Liang \\ Brain Research Center, University System of Taiwan, Taipei 112, Taiwan \\ Department of Biological Science and Technology, National Chiao-Tung University, Hsinchu 300, Taiwan \\ Email:sfliang@mail.nctu.edu.tw

\section{Teng-Yi Huang} \\ Brain Research Center, University System of Taiwan, Taipei 112, Taiwan \\ Department of Electrical and Control Engineering, National Chiao-Tung University, Hsinchu 300, Taiwan \\ Email: tyhuang.ece91g@nctu.edu.tw
}

Received 12 February 2004; Revised 14 March 2005

\begin{abstract}
The growing number of traffic accidents in recent years has become a serious concern to society. Accidents caused by driver's drowsiness behind the steering wheel have a high fatality rate because of the marked decline in the driver's abilities of perception, recognition, and vehicle control abilities while sleepy. Preventing such accidents caused by drowsiness is highly desirable but requires techniques for continuously detecting, estimating, and predicting the level of alertness of drivers and delivering effective feedbacks to maintain their maximum performance. This paper proposes an EEG-based drowsiness estimation system that combines electroencephalogram (EEG) log subband power spectrum, correlation analysis, principal component analysis, and linear regression models to indirectly estimate driver's drowsiness level in a virtual-reality-based driving simulator. Our results demonstrated that it is feasible to accurately estimate quantitatively driving performance, expressed as deviation between the center of the vehicle and the center of the cruising lane, in a realistic driving simulator.
\end{abstract}

Keywords and phrases: drowsiness, EEG, power spectrum, correlation analysis, linear regression model.

\section{INTRODUCTION}

Driving safety has received increasing attention due to the growing number of traffic accidents in recent years. Driver's fatigue has been implicated as a causal factor in many accidents. The National Transportation Safety Board found that 58 percent of 107 single-vehicle roadway departure crashes were fatigue-related in 1995, where the truck driver survived and no other vehicle was involved. Accidents caused by drowsiness at the wheel have a high fatality rate because of the marked decline in the driver's abilities of perception, recognition, and vehicle control abilities while sleepy. Preventing such accidents is thus a major focus of efforts in the field of active safety research $[1,2,3,4,5,6]$. A well-designed active safety system might effectively avoid accidents caused by drowsiness at the wheel. Many factors could contribute 
to drowsiness or fatigue, such as long working hours, lack of sleep, or the use of medication. Besides, another important factor of drowsiness is the nature of the task, such as monotonous driving on highways. The continued construction of highway and improvement of vehicle equipments have made it effortless for drivers to maneuver and operate their vehicles on the road for hours. An examination of the situations when drowsiness occurred shows that most of the accidents were on highways [4].

A number of methods have been proposed to detect vigilance changes in the past. These methods can be categorized into two main approaches. The first approach focuses on physical changes during fatigue, such as the inclination of the driver's head, sagging posture, and decline in gripping force on steering wheel $[7,8,9,10,11,12]$. These methods can be further classified as being either contact or else noncontact types in terms of the ways physical changes are measured. The contact type involves the detection of driver's movement by direct sensor contacts, such as using a cap or eyeglasses or attaching sensors to the driver's body. The noncontact type makes use of optical sensors or video cameras to detect vigilance changes. These methods monitor driving behavior or vehicle operation to detect driver fatigue. Driving behavior includes the steering wheel, accelerator, and brake pedal or transmission shift level, and the operation of vehicle includes the vehicle speed, lateral acceleration, and yaw rate or lateral displacement. Since these parameters vary in different vehicle types and driving conditions, it would be necessary to devise different detection logic for different types of vehicles.

The second approach focuses on measuring physiological changes of drivers, such as eye activity measures, heart beat rate, skin electric potential, and particularly, electroencephalographic (EEG) activities as a means of detecting the cognitive states $[13,14,15,16,17,18,19,20,21,22,23$, $24,25]$. Stern et al. $[22,23]$ reported that the eye blink duration and blink rate typically increase while blink amplitude decreases as function of the cumulative time on tasks. Other electrooculographic (EOG) studies have found that saccade frequencies and velocities decline as time on the task increases [24, 25]. Recently, Van Orden et al. [14]. further compared the eye-activity-based methods to EEGbased methods for alertness estimates in a compensatory visual tracking task. However, although these eye-activity variables are well correlated with the subject performance, those eye-activity-based methods require a relatively long moving-averaged window aiming to track slow changes in vigilance, whereas the EEG-based method can use a shorter moving-averaged window to track second-to-second fluctuations in the subject performance in a visual compensatory task $[14,15,16,17,18]$.

While approaches based on EEG signals have the advantages for making accurate and quantitative judgments of alertness levels, most recent psychophysiological studies have focused on using the same estimator for all subjects $[21,26,27]$. These methods did not account for large individual variability in EEG dynamics accompanying loss of alertness, and thus could not accurately estimate or predict individual changes in alertness and performance. In contrast,
Makeig and Inlow used individualized multiple linear regression models to estimate operators' changing levels of alertness [18]. Jung et al. further use the neural network model, applied to EEG power spectrum, in an auditory monitoring task and showed that a continuous, accurate, noninvasive, and near real-time estimation of an operator's global level of alertness is feasible $[15,16]$.

The scope of the current study is to examine neural activity correlates of fatigue/drowsiness in a realistic working environment. Our research investigates the feasibility of using multichannel EEG data to estimate and predict noninvasively the continuous fluctuations in human global-level alertness indirectly by measuring the driver's driving performance expressed as deviation between the center of the vehicle and the center of the cruising lane, in a very realistic driving task. To investigate the relationship of minute-scale fluctuations in performance to concurrent changes in the EEG spectrum, we first computed the correlations between changes in EEG power spectrum and the fluctuations in driving performance. We then build an individualized linear regression model for each subject applied to principal components of EEG spectra to assess the EEG dynamics accompanying loss of alertness for each operator. This approach can be used to construct and test a portable embedded system for a real-time alertness-monitoring system.

This paper is organized as follows. Section 2 describes the detailed descriptions of the EEG-based drowsiness experimental setup including the virtual-reality-based highway scene, subject instructions, physiological data collection, and alertness measurement. Detailed signal analysis of the collected data is given in Section 3. In Section 4, we explore the relationship between the alertness level, expressed as the driving performance, and the EEG power spectrum. Behavioral data are used to evaluate estimation performance of our alertness-monitoring model. Finally, we conclude our findings in Section 5.

\section{EXPERIMENTAL SETUP}

\subsection{Virtual-reality-based highway driving simulator}

In this study, we developed a VR-based 3D interactive highway scene using the high-fidelity emulation software, Coryphaeus, running on a high-performance SGI workstation. First, we created models of various objects (such as cars, roads, and trees, etc.) for the scene and setup the corresponding positions, attitudes, and other relative parameters between objects. Then, we developed the dynamic models among these virtual objects and built a complete highway simulated scene of full functionality with the aid of the high-level C-based API program. Figure 1 shows the VRbased highway scene displayed on a color XVGA 15" monitor (304.1 mm wide and $228.1 \mathrm{~mm}$ high) including four lanes from left to right, separated by a median stripe to simulate the view of the driver. The distance from the left-hand side to the right-hand side of the road is evenly divided into 256 parts (digitized into values $0-255$ ). The highway scene 


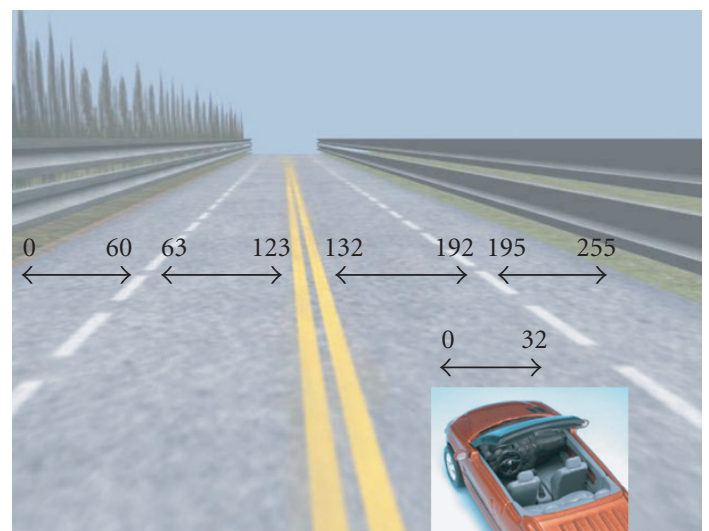

FIGURE 1: VR-based highway scene used in our experiments. The distance from the left side to the right side of the road is evenly divided into 256 parts (digitized into values $0-255$ ). The width of each lane is 60 units. The width of the car is 32 units. The refresh rate of highway scene was set properly to emulate a car driving at $100 \mathrm{~km} / \mathrm{h}$ fixed speed on the highway.

changes interactively as the driver/subject is driving the car at a fixed velocity of $100 \mathrm{~km} / \mathrm{hr}$ on the highway. The car is constantly and randomly drifted away from the center of the cruising lane, mimicking the consequences of a nonideal road surface. The highway scene was connected to a 36-channel physiological measuring system, where the EEG, EOG, ECG, and subject's performance, deviations between the center of the vehicle and the center of the cruising (third) lane, were continuously and simultaneously measured and recorded.

\subsection{Subjects}

Statistical reports [4] showed that the drowsiest time occurs from late night to early morning, and during the early afternoon hours. During these periods, drowsiness often occurs within one hour of continuous driving, indicating that drowsiness is not necessarily caused by long driving hours. Thus, the best time for doing the highway-drowsiness simulation is the early afternoon hours after lunch because drivers usually get drowsy within an hour of continuous driving. A total of ten subjects (ages from 20 to 40 years) participated in the VR-based highway driving experiments. Each subject completed simulated driving sessions on two separated days. On the first day, these participants were told of the general features of the driving task, completed necessary informed consent material, and then started with a $15 \sim 45$ minute practice to keep the car at the center of the cruising lane by maneuvering the car with the steering wheel. Subjects reported this amount of practice to be sufficient to train participants to asymptote on the task. After practicing, participants were then prepared with 33 EEG (including 2 EOG) electrodes referenced to the right earlobe based on a modified international 10-20 system, and 2 ECG electrodes placed on the chest. After a brief calibration procedure, subjects began a $\sim 45$ minute lane-keeping driving task and his/her EEG signals and driving performance defined as deviations of the center of the car from the center of the third lane of the road were measured and recorded simultaneously. Participants returned on a different day to complete the other $\sim 45$ min driving session. Participants who demonstrated waves of drowsiness involving two or more microsleeps in both sessions were selected for further analysis. Based on these criteria, five participants (10 sessions) were selected for further modeling and cross-session testing.

\subsection{Data collection}

During each driving session, 33 EEG/EOG channels (using sintered $\mathrm{Ag} / \mathrm{AgCl}$ electrodes), 2 ECG channels (bipolar connection), and the deviation between the center of the vehicle and the center of the cruising lane are simultaneously recorded by the Scan NuAmps Express system (Compumedics Ltd., VIC, Australia). Before data acquisition, the contact impedance between EEG electrodes and cortex was calibrated to be less than $5 \mathrm{k} \Omega$. The EEG data were recorded with 16-bit quantization level at a sampling rate of $500 \mathrm{~Hz}$ and then resampled down to $250 \mathrm{~Hz}$ for the simplicity of data processing.

\subsection{Alertness measurement}

To find the relationship between the measured EEG signals and the subject's cognitive state, and to quantify the level of the subject's alertness, we defined a subject's driving performance index as the deviation between the center of the vehicle and the center of the cruising lane. When the subject is drowsy (checked from video recordings), the value of driving performance index increases, and vice versa. The recorded driving performance time series were then smoothed using a causal 90-second square moving-averaged filter advancing at 2 -second steps to eliminate variance at cycle lengths shorter than 1-2 minutes since the fluctuates of drowsiness level with cycle lengths were in general longer than 4 minutes $[15,16]$.

\section{DATA ANALYSIS}

The flowchart of data analysis for estimating the level of alertness based on the EEG power spectrum was shown in Figure 2. For each subject, after collecting 33-channel EEG signals and driving deviations in a 45-minute simulated driving session, the EEG data were first preprocessed using a simple lowpass filter with a cut-off frequency of $50 \mathrm{~Hz}$ to remove the line noise and other high-frequency noise. Then, we calculated the moving-averaged log power spectra of all $33 \mathrm{EEG}$ channels. The correlation coefficients between the smoothed subjects' driving performance and the log power spectra of all EEG channels at each frequency band are further evaluated to form a correlation spectrum. The log power spectra of 2 EEG channels with the highest correlation coefficients are further decomposed using principal component analysis (PCA) algorithm to reduce feature dimensions. Then the first 50 representative PCA components with higher eigenvalues were selected as the input vectors of the linear regression model to estimate the individual subject's driving performance. Detailed analyses are described in the following subsections. 


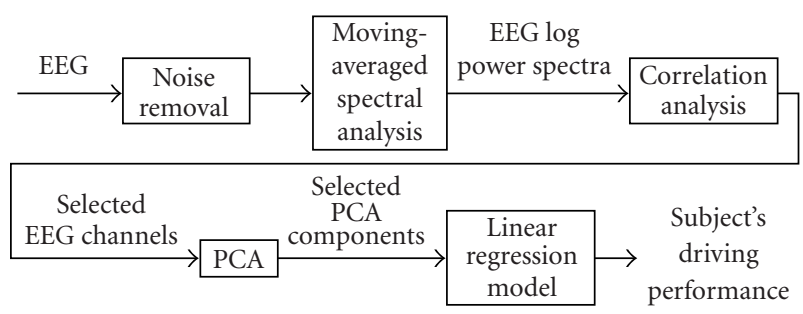

FIGURE 2: Flowchart for processing the EEG signals. (1) A lowpass filter was used to remove the line noise and higher-frequency ( $>50 \mathrm{~Hz}$ ) noise. (2) Moving-averaged spectral analysis was used to calculate the EEG log power spectrum of each channel advancing at 2-second steps. (3) Two EEG channels with higher correlation coefficients between subject's driving performance and EEG log power spectrum were further selected. (4) Principal component analysis was trained and used to decompose selected features and extract the representative PCA-components as the input vectors for the linear regression models. (5) The linear regression models were trained in one training session and used to continuously estimate and predict the individual subject's driving performance in the testing session.

\subsection{Moving-averaged power spectral analysis}

Moving-averaged spectral analysis of the EEG data as shown in Figure 3 was first accomplished using a 750-point Hanning window with 250-point overlap. Windowed 750-point epochs were further subdivided into several 125-point subwindows using the Hanning window again with 25-point steps, each extended to 256 points by zero padding for a 256 point FFT. A moving median filter was then used to average and minimize the presence of artifacts in the EEG records of all subwindows. The moving-averaged EEG power spectra were further converted into a logarithmic scale for spectral correlation and driving performance estimation $[28,29]$. Thus, the time series of EEG $\log$ power spectrum for each session consisted of 33-channel EEG power spectrum estimated across 40 frequencies (from 1 to $40 \mathrm{~Hz}$ ) stepping at 2-second (500-point, an epoch) time intervals.

\subsection{Correlation analysis}

Since alertness level fluctuates with cycle lengths longer than 4 minutes $[15,16]$, we smoothed the EEG power and driving performance time series using a causal 90 -second square moving-averaged filter to eliminate variances at cycle lengths shorter than 1-2 minutes. To investigate the relationship of minute-scale fluctuations in continuous driving performance with concurrent changes in the 33-channel EEG power spectrum over times and subjects, we measured correlations between changes in the EEG log power spectrum and driving performance as forming a correlation spectrum by computing the Pearson's correlation coefficients between two time series at each EEG frequency expressed as $\operatorname{Corr}_{x y}=\left(\sum(x-\bar{x}) *(y-\bar{y})\right) / \sqrt{\sum(x-\bar{x})^{2} * \sum(y-\bar{y})^{2}}$. The channels with higher correlated coefficients between the EEG $\log$ power spectrum and the subject driving performance were further selected (see Section 4.1), and the dimensions

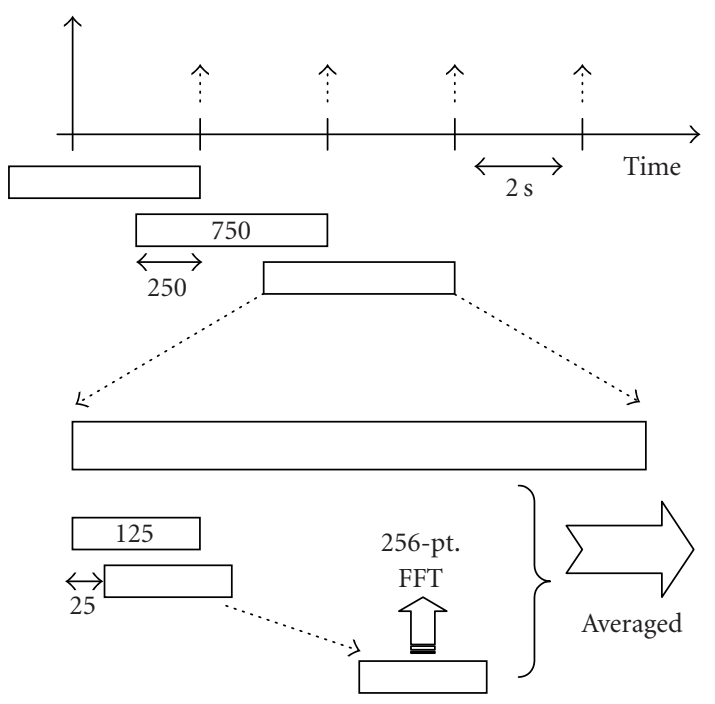

FIGURE 3: Block diagram for moving-averaged spectral analysis. The EEG data was first divided using a 750-point Hanning window with 250-point overlap. The 750-point epochs were further divided into several 125-point frames using Hanning windows again with 25point step size, and each frame was applied for a 256-point FFT by zero padding. Then the subwindow power spectrum was further averaged and converted to a logarithmic scale to form a log power spectrum.

of selected EEG power spectrum of such channels were reduced using principal component analysis (PCA) algorithm.

\subsection{Feature extraction}

In this study, we use a multivariate linear regression model [30] to estimate/predict the subject's driving performance based on the information available in the EEG log power spectrum at sites $\mathrm{Cz}$ and $\mathrm{Pz}$ (as suggested in Section 4.1). The EEG power spectrum time series for each session consisted of 1350 (750-point, an epoch) EEG power estimates at 40 frequencies (from 1 to $40 \mathrm{~Hz}$ ) at 2-5 time intervals. We then applied Karhunen-Loeve principal component analysis (PCA) to the full EEG log spectrum to decompose the EEG log power spectrum time series and extract the directions of the largest variance for each session. The PCA is a linear transformation that can find the principal coordinate axes of samples such that along the new axes, the sample variances are extremes (maxima and minima) and uncorrelated. Using a cutoff on the spread along each axis, a sample may thus be reduced in its dimensionality [31]. The principal axes and the variance along each of them are given by the eigenvectors and associated eigenvalues of the dispersion matrix. In our study, the projections of the PCA components accounting for the largest 50 eigenvalues were then used as inputs to train the individual linear regression models for each subject, which used a 50-order linear polynomial with a least-squareerror cost function to estimate the time course of the driving performance. Each model was trained using the features only extracted in the training session and tested on a separate testing session of the same subject for each of the five 
selected subjects. The parameters of PCA (eigenvectors) from the training sessions were used to project features in the testing sessions so that all data were processed in the same way for the same subject before feeding to the estimation models.

\section{RESULTS AND DISCUSSION}

\subsection{Relationship between the EEG spectrum and subject alertness}

To investigate the relationship of minute-scale fluctuations in driving performance to concurrent changes in the EEG spectrum, we measured correlations between changes in the EEG power spectrum and driving performance by computing the correlation coefficients between the two time series at each EEG frequency. We refer to the results as forming a correlation spectrum. For each EEG site and frequency, we then computed spectral correlations for each session separately and averaged the results across all 10 sessions from the five subjects. Figure $4 a$ shows the results for 40 frequencies between 1 and $40 \mathrm{~Hz}$. Note that the mean correlation between performance and EEG power is predominantly positive at all EEG channels below $20 \mathrm{~Hz}$. We also investigated the spatial distributions of these positive correlations by plotting the correlations between EEG power spectrum and driving performance, computed separately at dominant frequency bins $7,12,16$, and $20 \mathrm{~Hz}$ (cf. Figure $4 \mathrm{a}$ ) on the scalp (Figure $4 \mathrm{~b}$ ). As the results in Figure $4 \mathrm{a}$ show, the correlation coefficients plotted on the scalp maps are predominantly positive. The correlations are particularly strong at central and posterior channels, which are similar to the results of previous studies in the driving experiments [21, 26, 27]. The relatively high correlation coefficients of EEG log power spectrum with driving performance suggest that using EEG log power spectrum may be suitable for drowsiness (microsleep) estimation, where the subject's cognitive state might fall into stage one of the nonrapid eye movement (NREM) sleep. To be practical for routine use during driving or in other occupations, EEG-based cognitive assessment systems should use as few EEG sensors as possible to reduce the preparation time for wiring drivers and computational load for estimating continuously the level of alertness in near real time. According to the correlations shown in Figure $4 \mathrm{~b}$, we believe it is adequate to use the EEG signals at sites $\mathrm{Cz}$ and $\mathrm{Pz}$ to assess the alertness level of drivers continuously.

Next, we compared correlation spectra for individual sessions to examine the stability of this relationship over time and subjects. Figures 5 and 6 plot correlation spectra at sites $\mathrm{Fz}, \mathrm{Cz}, \mathrm{Pz}$, and $\mathrm{Oz}$ of two separate driving sessions for extreme cases from Subjects A (best) and B (worst), respectively. The relationship between EEG power spectrum and driving performance is stable within the subjects, especially below $20 \mathrm{~Hz}$. However, the relationship is variable from subject to subject (compare Figures 5 and 6 ). The time intervals between the training and testing sessions of the lane-keeping experiments ranged from one day to one week long for the selected five subjects.

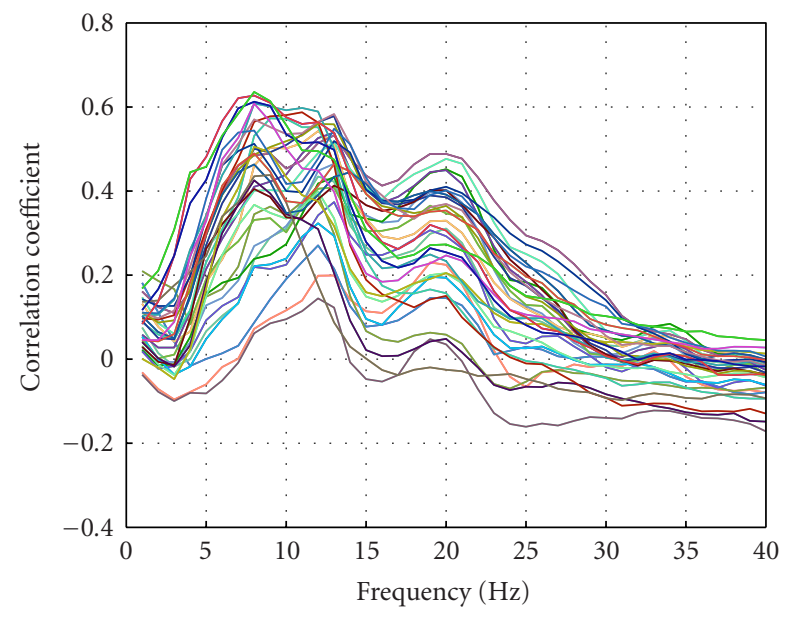

(a)
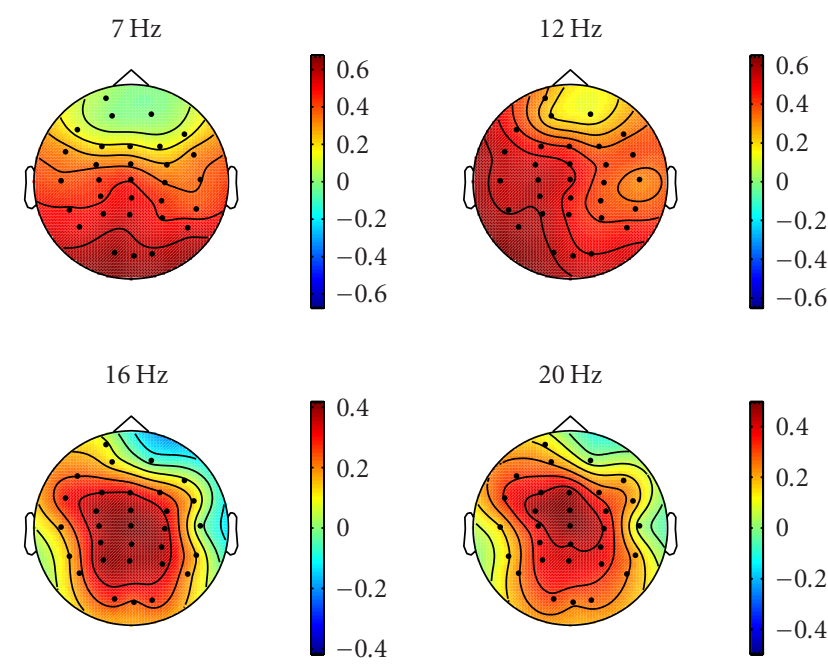

(b)

FIgURE 4: Correlation spectra. Correlations between EEG power and driving performance, computed separately for 40 EEG frequencies between 1 and $40 \mathrm{~Hz}$. (a) Grand mean correlation spectra for 10 sessions on 5 subjects. (b) Scalp topographies of the correlations at dominant frequencies at 7, 12, 16, and $20 \mathrm{~Hz}$.

The above analyses provide strong and converging evidence that changes in subject's alertness level indexed by driving performance during a driving task are strongly correlated with the changes in the EEG power spectrum at several frequencies at central and posterior sites. This relationship is relatively variable between subjects, but stable within subjects, consistent with the findings from a simple auditory target detection task reported in $[15,16]$. These findings suggest that information available in the EEG can be used for real-time estimation of changes in alertness of human operators performing monitoring tasks. However, for maximal accuracy the estimation algorithm should be capable of adapting to individual differences in the mapping between EEG and alertness. 


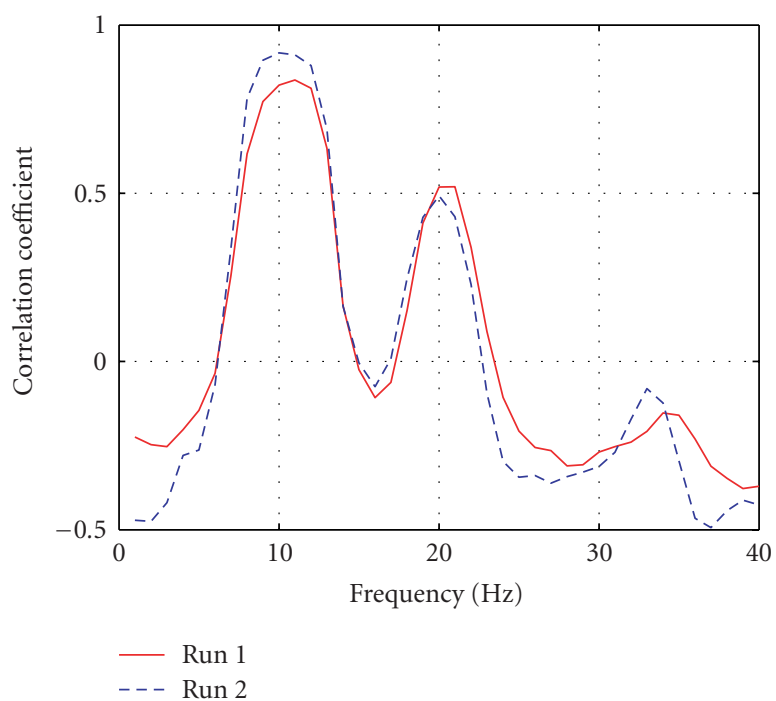

(a)

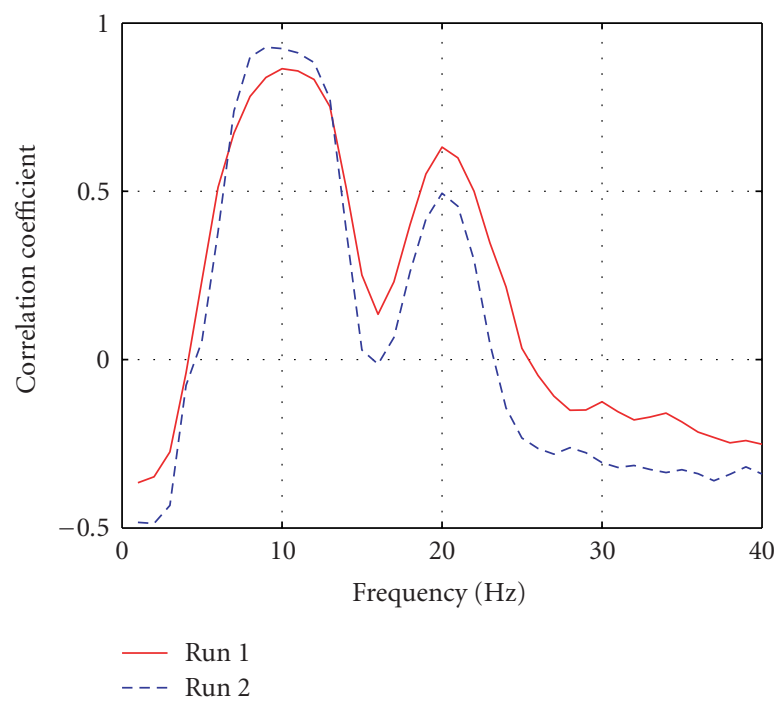

(c)

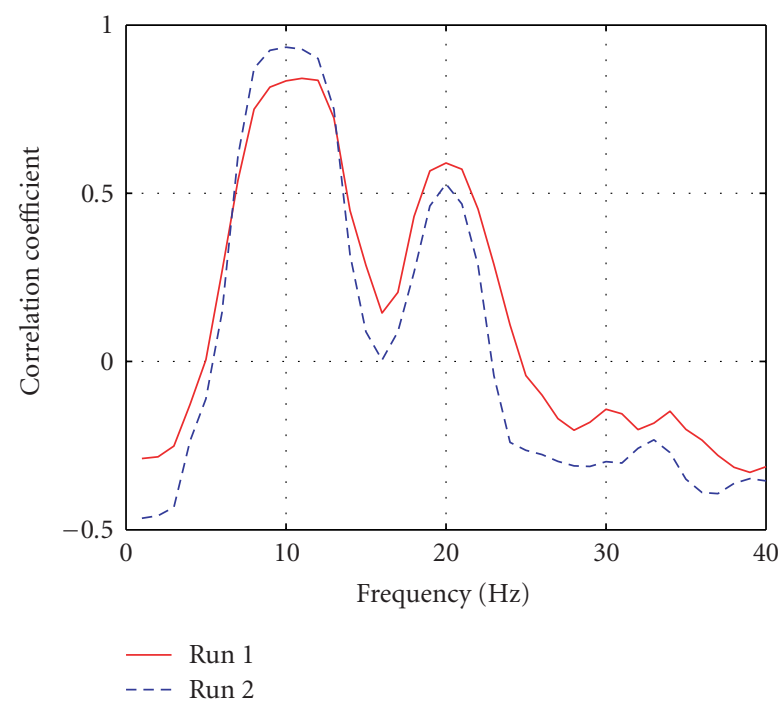

(b)

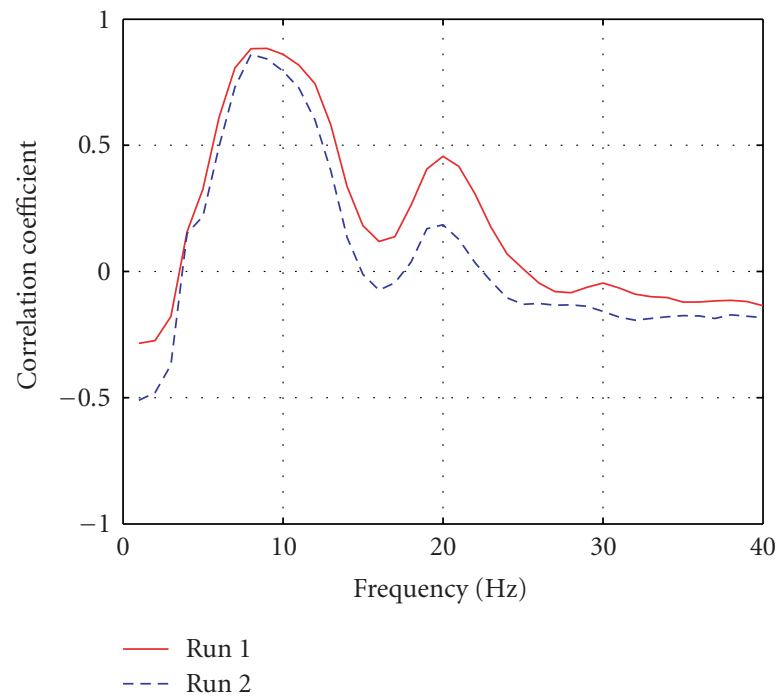

(d)

Figure 5: Correlation spectra between the EEG power spectrum and the driving performance at (a) Fz, (b) Cz, (c) Pz, and (d) Oz channels in two separate driving sessions for Subject A (best case). Note that the relationship between the EEG power spectrum and the driving performance is stable within this subject.

\subsection{EEG-based driving performance estimation/prediction}

In order to estimate/predict the subject's driving performance based on the information available in the EEG power spectrum at sites $\mathrm{Cz}$ and $\mathrm{Pz}$, a 50-order linear regression model $y=\sum_{i=1}^{N=50} a_{i} x_{i}+a_{0}$ with a least-square-error cost function is used, where $y$ is the desired output, $x$ is the input feature, $N$ is the order ( $N=50$ in this case), $a_{i}$ 's are the parameters, and $a_{0}=1$ is the constant. We used only two EEG channels $(\mathrm{Cz}$ and $\mathrm{Pz})$ that showed the highest correlation between the EEG power spectrum and the driving performance because using all 33 channels may introduce more unexpected noise. Figure 7 plots the estimated and actual driving performance of a session of Subject A. The linear regression model in this figure is trained with and tested against the same session, that is, within-session testing. As can been seen, the estimated driving performance matched extremely well the actual driving performance $(r=0.88)$. When the model was tested against a separate test session of the same subject as shown in Figure 8, the correlation between the actual and estimated driving performance, though decreased, remained high $(r=0.7)$. Across ten sessions, the mean correlation coefficient between actual driving performance time series and within-session estimation is $0.90 \pm 0.034$, whereas the mean correlation coefficient between actual driving performance and cross-session estimation is $0.53 \pm 0.116$. These results suggest that continuous EEG-based driving 


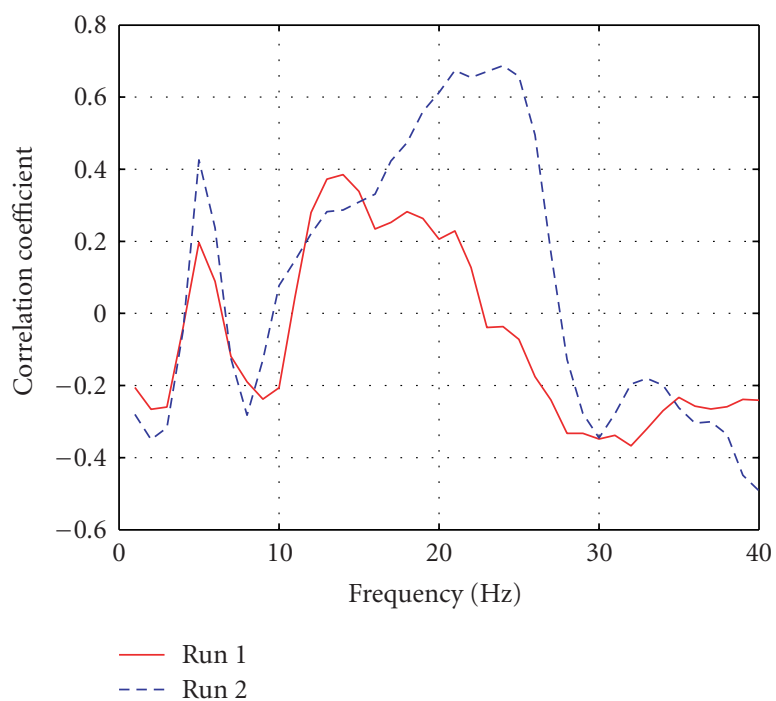

(a)

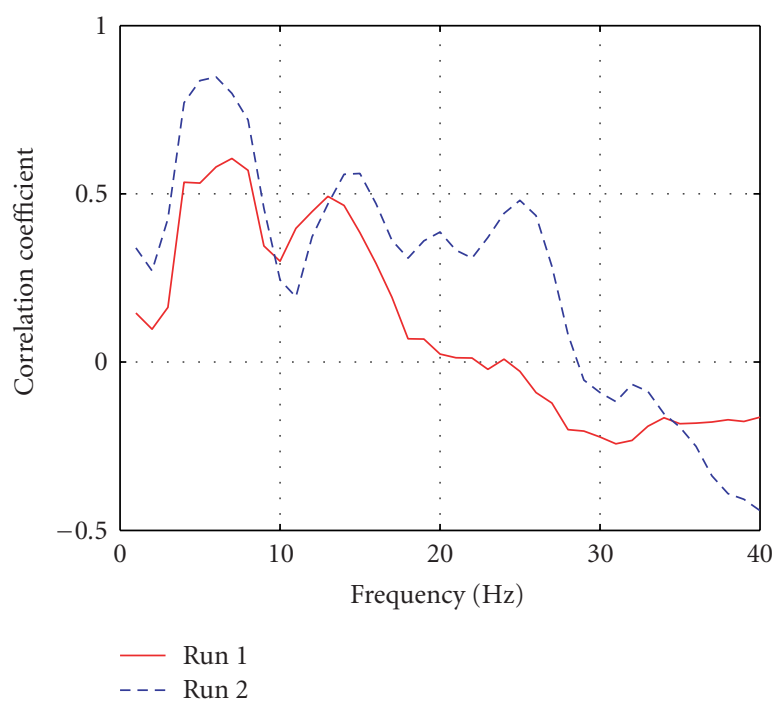

(c)

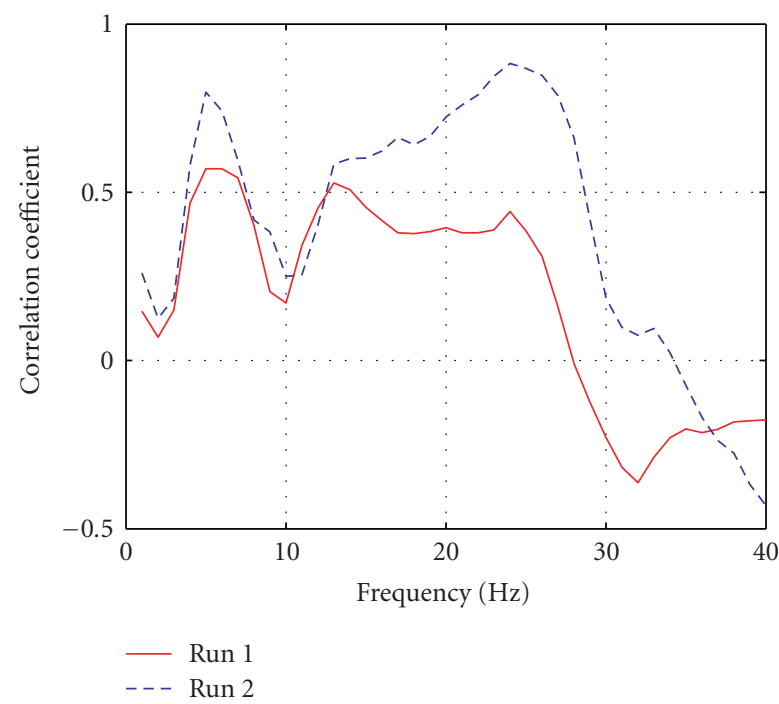

(b)

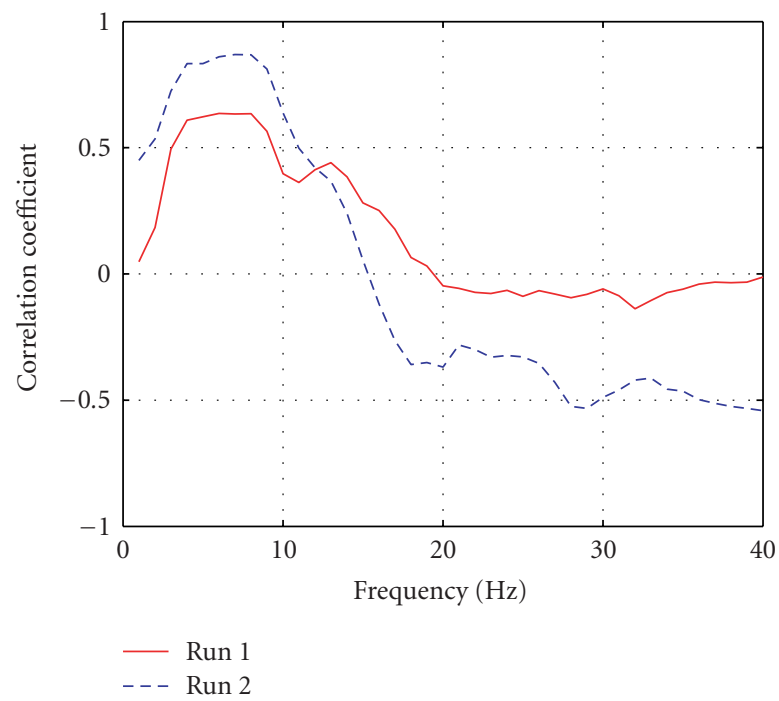

(d)

FIgure 6: Correlation spectra between the EEG power spectrum and the driving performance at (a) Fz, (b) Cz, (c) Pz, and (d) Oz channels in two separate driving sessions for Subject B (worst case). Note that the relationship between the EEG power spectrum and the driving performance is stable within this subject, especially below $20 \mathrm{~Hz}$. However, the relationship is variable from subject to subject (compare Figures 5 and 6).

performance estimation using a small number of data channels is feasible, and can give accurate information about minute-to-minute changes in operator alertness.

\section{CONCLUSIONS}

In this study, we demonstrated a close relationship between minute-scale changes in driving performance and the EEG power spectrum. This relationship appears stable within individuals across sessions, but is somewhat variable between subjects. We also combined EEG power spectrum estimation, correlation analysis, PCA, and linear regression to continuously estimate/predict fluctuations in human alertness level indexed by driving performance measurement, deviation between the center of the vehicle and the center of the cruising lane. Our results demonstrated that it is feasible to accurately estimate driving errors based on multi-channel EEG power spectrum estimation and principal component analysis algorithm. The computational methods we employed in this study were well within the capabilities of modern real-time embedded digital signal processing hardware to perform in real time using one or more 


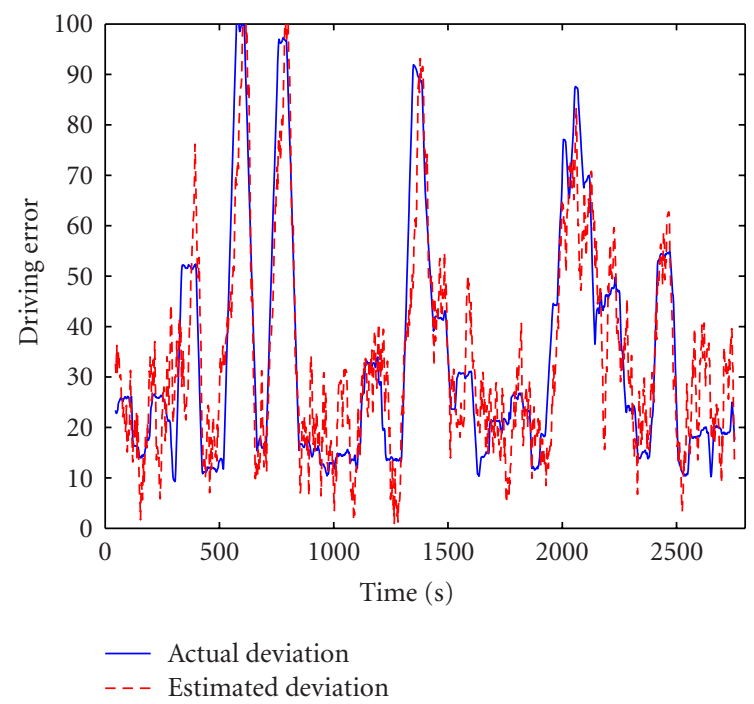

Figure 7: Driving performance estimates for a session of Subject A, based on a linear regression (dashed line) of PCA-reduced EEG log spectra at two scalp sites, overplotted against actual driving performance time series for the session (solid line). The correlation coefficient between the two time series is $r=0.88$.

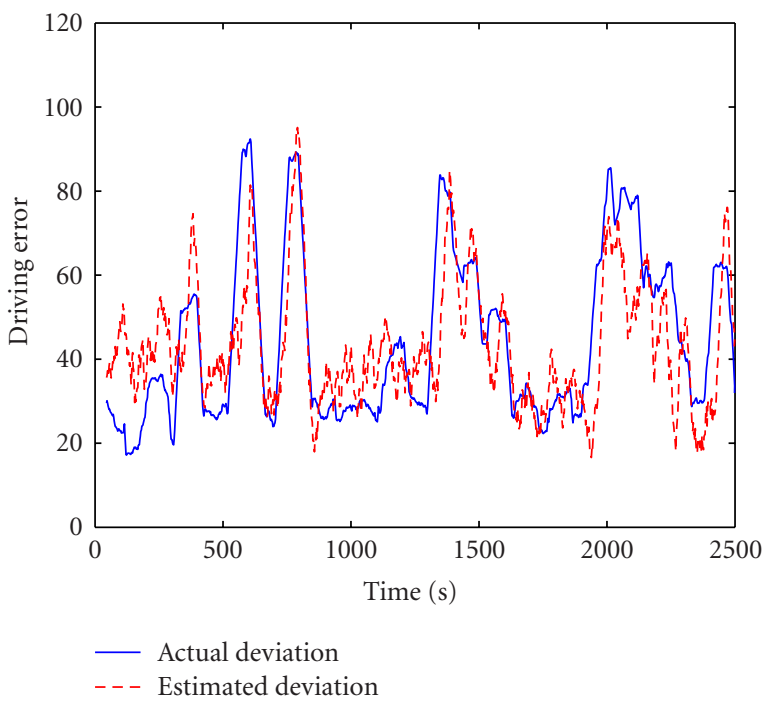

FIGURE 8: Driving performance estimates for a test session, based on a linear regression (dashed line) of PCA-reduced EEG log spectra from a separate training session of the same subject, overplotted against actual driving performance time series of the test session (solid line). The correlation coefficient between the two time series is $r=0.7$. Note that the training and testing data in this study were completely disjoined.

channels of EEG data. Once an estimator has been developed for each driver, based on limited pilot testing, the method uses only spontaneous EEG signals from the individual, and does not require further collection or analysis of operator performance. The proposed methods thus might be used to construct and test a portable embedded system for a realtime alertness-monitoring system.

\section{ACKNOWLEDGMENTS}

The authors would like to thank Mrs. Jeng-Ren Duann, Chun-Fei Hsu, Wen-Hung Chao, Yu-Chieh Chen, KuanChih Huang, Shih-Cheng Guo, and Yu-Jie Chen for their great help in developing and operating the experiments. This work was supported in part by the Ministry of Education, Taiwan, under Grant EX-91-E-FAOE-4-4 and Ministry of Economic Affairs, Taiwan, under Grant 93-17-A-02-S1-032 to C. T. Lin and associates and a Grant from Swartz Foundation to T. P. Jung.

\section{REFERENCES}

[1] J. French, "A model to predict fatigue degraded performance," in Proc. IEEE 7th Conference on Human Factors and Power Plants, vol. 4, pp. 6-9, Scottsdate, Ariz, USA, September 2002.

[2] W. W. Wierwille, S. S. Wreggit, and R. R. Knipling, "Development of improved algorithms for on-line detection of driver drowsiness," in Proc. Convergence '94, International Congress on Transportation Electronics, SAE (Society of Automotive Engineers), pp. 331-340, Detroit, Mich, USA, October 1994.

[3] A. Amditis, A. Polychronopoulos, E. Bekiaris, and P. C. Antonello, "System architecture of a driver's monitoring and hypovigilance warning system," in Proc. IEEE Intelligent Vehicle Symposium (IV '02), vol. 2, pp. 527-532, Versailles, France, June 2002.

[4] H. Ueno, M. Kaneda, and M. Tsukino, "Development of drowsiness detection system," in Proc. Vehicle Navigation and Information Systems Conference (VNIS '94), pp. 15-20, Yokohama, Japan, August-September 1994.

[5] R. Grace, V. E. Byrne, D. M. Bierman, et al., "A drowsy driver detection system for heavy vehicles," in Proc. AIAA/IEEE/SAE 17th Conference on Digital Avionics Systems (DASC'98), vol. 2, pp. I36/1-I36/8, Bellevue, Wash, USA, October-November 1998.

[6] T. Pilutti and A. G. Ulsoy, "Identification of driver state for lane-keeping tasks," IEEE Trans. Syst., Man, Cybern. A, vol. 29, no. 5, pp. 486-502, 1999.

[7] P. Smith, M. Shah, and N. da Vitoria Lobo, "Monitoring head/eye motion for driver alertness with one camera," in Proc.15th International Conference on Pattern Recognition (ICPR '00), vol. 4, pp. 636-642, Barcelona, Spain, September 2000.

[8] C. M. Frederick-Recascino and M. Hilscher, "Monitoring automated displays: effects of and solutions for boredom," in Proc. 20th Conference of Digital Avionics Systems (DASC '01), vol. 1, pp. 5D3/1-5D3/5, Daytona Beach, Fla, USA, October 2001.

[9] G. Kaefer, G. Prochart, and R. Weiss, "Wearable alertness monitoring for industrial applications," in Proc. 7th IEEE International Symposium on Wearable Computers (ISWC '03), pp. 254-255, White Plains, NY, USA, October 2003.

[10] K. B. Khalifa, M. H. Bedoui, R. Raytchev, and M. Dogui, "A portable device for alertness detection," in Proc. 1st Annual International IEEE-EMBS Special Topic Conference on Microtechnologies in Medicine \& Biology, pp. 584-586, Lyon, France, October 2000.

[11] C. A. Perez, A. Palma, C. A. Holzmann, and C. Pena, "Face and eye tracking algorithm based on digital image processing," in Proc. IEEE International Conference on Systems, Man, and Cybernetics (SMC'01), vol. 2, pp. 1178-1183, Tucson, Ariz, USA, October 2001.

[12] J. C. Popieul, P. Simon, and P. Loslever, "Using driver's head movements evolution as a drowsiness indicator," in Proc. IEEE 
International Intelligent Vehicles Symposium (IV'03), pp. 616621, Columbus, Ohio, USA, June 2003.

[13] T. L. Morris and J. C. Miller, "Electrooculographic and performance indices of fatigue during simulated flight," Biological Psychology, vol. 42, no. 3, pp. 343-360, 1996.

[14] K. Van Orden, W. Limbert, S. Makeig, and T.-P. Jung, "Eye activity correlates of workload during a visualspatial memory task," Human Factors, vol. 43, no. 1, pp. 111-121, 2001.

[15] T.-P. Jung, S. Makeig, M. Stensmo, and T. J. Sejnowski, "Estimating alertness from the EEG power spectrum," IEEE Trans. Biomed. Eng., vol. 44, no. 1, pp. 60-69, 1997.

[16] S. Makeig and T.-P. Jung, "Changes in alertness are a principal component of variance in the EEG spectrum," Neuroreport, vol. 7, no. 1, pp. 213-216, 1995.

[17] M. Matousek and I. Petersén, "A method for assessing alertness fluctuations from EEG spectra," Electroencephalography and Clinical Neurophysiology, vol. 55, no. 1, pp. 108-113, 1983.

[18] S. Makeig and M. Inlow, "Lapses in alertness: coherence of fluctuations in performance and EEG spectrum," Electroencephalography and Clinical Neurophysiology, vol. 86, no. 1, pp. 23-35, 1993.

[19] J. Qiang, Z. Zhiwei, and P. Lan, "Real-time nonintrusive monitoring and prediction of driver fatigue," IEEE Trans. Veh. Technol., vol. 53, no. 4, pp. 1052-1068, 2004.

[20] S. Makeig and T.-P. Jung, "Tonic, phasic, and transient EEG correlates of auditory awareness in drowsiness," Cognitive Brain Research, vol. 4, no. 1, pp. 15-25, 1996.

[21] S. Roberts, I. Rezek, R. Everson, H. Stone, S. Wilson, and C. Alford, "Automated assessment of vigilance using committees of radial basis function analysers," IEE ProceedingsScienceMeasurement \& Technology, vol. 147, no. 6, pp. 333$338,2000$.

[22] J. A. Stern, D. Boyer, and D. Schroeder, "Blink rate: a possible measure of fatigue," Human Factors, vol. 36, no. 2, pp. 285297, 1994.

[23] J. A. Stern, L. C. Walrath, and R. Goldstein, "The endogenous eyeblink," Psychophysiology, vol. 21, no. 1, pp. 22-33, 1984.

[24] D. Schmidt, L. A. Abel, L. F. Dell'Osso, and R. B. Daroff, "Saccadic velocity characteristics: intrinsic variability and fatigue," Aviation, Space and Environmental Medicine, vol. 50, no. 4, pp. 393-395, 1979.

[25] D. K. McGregor and J. A. Stern, "Time on task and blink effects on saccade duration," Ergonomics, vol. 39, no. 4, pp. 649 660, 1996.

[26] B. J. Wilson and T. D. Bracewell, "Alertness monitor using neural networks for EEG analysis," in Proc. IEEE Signal Processing Society Workshop on Neural Networks for Signal Processing X, vol. 2, pp. 814-820, Sydney, NSW, Australia, December 2000.

[27] P. Parikh and E. Micheli-Tzanakou, "Detecting drowsiness while driving using wavelet transform," in Proc. IEEE 30th Annual Northeast on Bioengineering Conference, pp. 79-80, Boston, Mass, USA, April 2004.

[28] M. Steriade, "Central core modulation of spontaneous oscillations and sensory transmission in thalamocortical systems," Current Opinion in Neurobiology, vol. 3, no. 4, pp. 619-625, 1993.

[29] M. Treisman, "Temporal rhythms and cerebral rhythms," in Timing and Time Perception, J. Gibbon and L. Allen, Eds., vol. 423, pp. 542-565, New York Academy of Sciences, New York, NY, USA, 1984.

[30] S. Chatterjee and A. S. Hadi, "Influential observations, high leverage points, and outliers in linear regression," Statistical Science, vol. 1, no. 3, pp. 379-416, 1986.
[31] C. M. Bishop, Neural Networks for Pattern Recognition, Oxford University Press, Oxford, UK, 1995.

Chin-Teng Lin received the B.S. degree from the National Chiao-Tung University (NCTU), Taiwan, in 1986, and the Ph.D. degree in electrical engineering from Purdue University, USA, in 1992. He is currently the Chair Professor and Associate Dean of the College of Electrical Engineering and Computer Science, and Director of the Brain Research Center at NCTU. He is the author of Neural Fuzzy Systems (Pren-

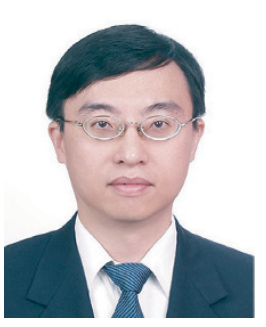
tice Hall). He has published about 90 journal papers including over 65 IEEE journal papers. He is an IEEE Fellow for his contributions to biologically inspired information systems. He serves on Board of Governors at the IEEE CAS and SMC Societies now. He has been the President of Asia Pacific Neural Network Assembly since 2004. He has received the Outstanding Research Award granted by the National Science Council, Taiwan, since 1997 to present, received the Outstanding Engineering Professor Award granted by the Chinese Institute of Engineering (CIE) in 2000, and the 2002 Taiwan Outstanding InformationTechnology Expert Award. He was also elected to be one of the 38th Ten Outstanding Rising Stars in Taiwan (2000). He currently serves as an Associate Editor of the IEEE Transactions on Circuits and Systems, Part I \& Part II, IEEE Transactions on Systems, Man, Cybernetics, IEEE Transactions on Fuzzy Systems, and so forth.

Ruei-Cheng Wu received the B.S. degree in nuclear engineering from the National Tsing-Hua University, Taiwan, in 1995, and the M.S. degree in control engineering from the National Chiao-Tung University, Taiwan, in 1997. He is currently pursuing the Ph.D. degree in electrical and control engineering at the National Chiao-Tung University, Taiwan. His current research interests are biomedical signal processing, multimedia signal processing, fuzzy neural networks, and linear control.

Tzyy-Ping Jung received the B.S. degree in electronics engineering from the National Chiao Tung University, Taiwan, in 1984, and the M.S. and Ph.D. degrees in electrical engineering from The Ohio State University in 1989 and 1993, respectively. He was a Research Associate at the National Research Council of the National Academy of Sciences and at the Computational Neurobiology Laboratory, The Salk Institute, San Diego, Calif. He is currently an Associate Research Professor at the Institute for Neural Computation of the University of California, San Diego. He is also the Associate Director of the Swartz Center for Computational Neuroscience at UCSD. His research interests are in the areas of biomedical signal processing, cognitive neuroscience, artificial neural networks, time-frequency analysis of human EEG, functional neuroimaging, and the development of neural humansystem interfaces.
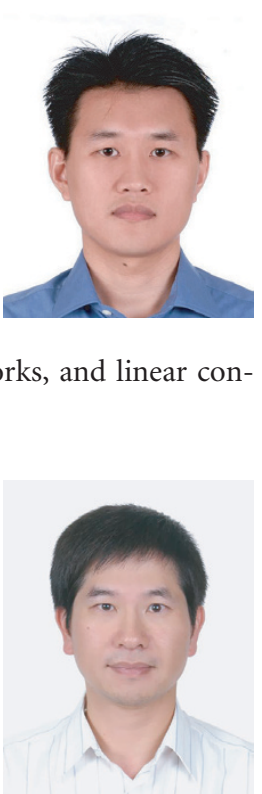
Sheng-Fu Liang was born in Tainan, Taiwan, in 1971. He received the B.S. and M.S. degrees in control engineering from the $\mathrm{Na}$ tional Chiao-Tung University (NCTU), Taiwan, in 1994 and 1996, respectively. He received the Ph.D. degree in electrical and control engineering from NCTU in 2000. From 2001 to 2005, he was a Research Assistant Professor in electrical and control engineering, NCTU. In 2005, he joined the De-

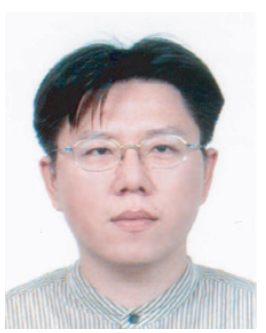
partment of Biological Science and Technology, NCTU, where he serves as an Assistant Professor. He has also served as the Chief Executive of the Brain Research Center, NCTU Branch, University System of Taiwan, since September 2003. His current research interests are biomedical engineering, biomedical signal/image processing, machine learning, fuzzy neural networks (FNN), the development of brain-computer interface (BCI), and multimedia signal processing.

Teng-Yi Huang received the B.S. degree in electrical engineering from the National Central University, Taiwan, in 2002, and the M.S. degree in electrical and control engineering from the National Chiao-Tung University, Taiwan, in 2004. He is currently pursuing the Ph.D. degree at the National Chiao-Tung University, Taiwan. His research interests are in the areas of biomedical signal processing, biofeedback control,

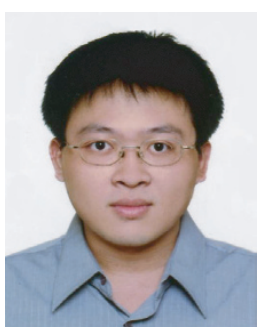
and virtual reality technology. 